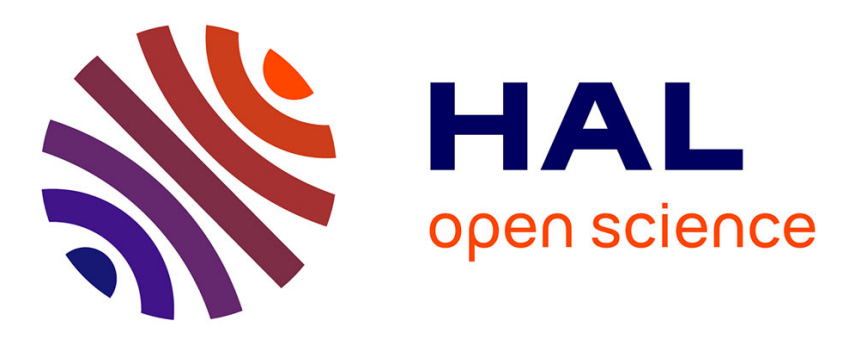

\title{
Use of the distance transform for the integration of local measurements : principle and application in the field of chemical engineering.
}

Loïc Sorbier, Frédéric Bazer-Bachi, Yannick Blouet, Maxime Moreaud, Virginie Moizan-Baslé

\section{To cite this version:}

Loïc Sorbier, Frédéric Bazer-Bachi, Yannick Blouet, Maxime Moreaud, Virginie Moizan-Baslé. Use of the distance transform for the integration of local measurements: principle and application in the field of chemical engineering.. Microscopy and Microanalysis, 2016, 22 (2), pp.422-431. 10.1017/S1431927616000015 . hal-01394873

\section{HAL Id: hal-01394873 \\ https://hal.science/hal-01394873}

Submitted on 2 Feb 2017

HAL is a multi-disciplinary open access archive for the deposit and dissemination of scientific research documents, whether they are published or not. The documents may come from teaching and research institutions in France or abroad, or from public or private research centers.
L'archive ouverte pluridisciplinaire HAL, est destinée au dépôt et à la diffusion de documents scientifiques de niveau recherche, publiés ou non, émanant des établissements d'enseignement et de recherche français ou étrangers, des laboratoires publics ou privés. 
Use of the Distance Transform for the Integration of Local Measurements: Principle and Application in the Field of Chemical Engineering

Brief Title : Integration by the Distance Transform

Loïc Sorbier, Frédéric Bazer-Bachi, Yannick Blouët, Maxime Moreaud, Virginie MoizanBasle

IFP Energies nouvelles, Rond-point de l'échangeur de Solaize, BP 3, 69360 Solaize, France

Corresponding author: Loïc Sorbier IFP Energies nouvelles, Rond-point de l'échangeur de Solaize, BP 3, 69360 Solaize, France. Tel: (+33) 4377029 69, Fax: (+33) 4377027 45, loic.sorbier@ifpen.fr 


\begin{abstract}
We propose an original methodology to integrate local measurement for non-trivial object shape. The method employs the distance transform of the object and least-square fitting of numerically computed weighting functions extracted from it. The method is exemplified in the field of chemical engineering by calculating the global metal concentration in catalyst grains from uneven metal distribution profiles. Applying the methodology on synthetic profiles with the help of a very simple deposition model allows us to evaluate the accuracy of the method. For high symmetry objects such as an infinite cylinder, relative errors on global concentration are found lower than $1 \%$ for well resolved profiles. For a less symmetrical object, a tetralobe, the best estimator gives a relative error below $5 \%$ at the cost of increased measurement time. The applicability on a real-case is demonstrated on an aged hydrodemetallation catalyst. Sampling of catalyst grains at the inlet and the outlet of the reactor let us conclude to different reactivity for the trapped metals.
\end{abstract}

\title{
Key words
}

Distance transform, Integration, EPMA, Catalyst, Element profile, Metal deposition. 


\section{Introduction}

It is sometimes necessary to convert local concentration measurements performed by Electron-Probe Micro Analysis (EPMA) or other spatial resolved techniques to global information at the sample scale. This need of an integration of local measurements instead of using directly global elemental techniques such as X-ray fluorescence (XRF) or inductively coupled plasma optical emission spectrometry (ICP-OES) may arise in three kind of situations. The first situation is when the sample is unique or with a too small quantity to be able to do both local and global analysis. This situation is often encountered in high throughput experiments where sample mass engaged is very low. The second situation is encountered for quality control of manufactured object. If both elemental repartition inside the object and global concentrations of elements in the object need to be checked, integration of local measurements acquired on several objects is the best way to obtain simultaneously both information. Last situation concerns criterions that are mixing local and global quantity or defined as integrals of local quantities.

Uneven element distribution profiles are encountered in chemical engineering, in catalysts under internal diffusional limitations, where reaction occurs quicker than reactant diffusion in the catalyst grain. Hydrodemetallation (HDM) of oil residues and selective hydrogenation of unsaturated hydrocarbon feedstock are two of the important industrial chemical processes working within the internal diffusional limitation regime. For these kind of catalysts, the concentration profile of uneven deposited elements in the grain has to be converted into a global information at the grain scale to provide descriptors related to catalyst performance. It may be the total quantity of an element in a grain to check the homogeneity of production batches (Sorbier, 2013), the thickness of the element deposit (Sorbier et al. 2012) or a measure of the catalyst efficiency (Tamm et al. 1981). 
HDM of oil residues has an important and growing part due to the rarefaction of light crudes, increasing demand for middle distillates and harsher specification on sulfur contents of the end products (Merdrignac et al., 2013). For catalyst employed in HDM of oil residues, the ratio of the global vanadium concentration in the pellet to the vanadium concentration at the border of the pellet gives the catalyst efficiency (Tamm et al. 1981). Measurements on several grains using only a local technique are required if one wants to check the homogeneity of catalytic efficiency at the reactor scale. Selective hydrogenation is important for the purification of feedstock before polymer synthesis (polyethylene, polypropylene, polystyrene, ...). For selective hydrogenation palladium catalysts, the thickness of the palladium deposit is correlated with the catalyst apparent efficiency (Sorbier et al. 2012). This thickness is defined as the distance to the border of the pellet containing a prescribed proportion (for example 98\%) of the global element content. Considering these two examples of catalysts, a proper strategy for local measurement integration is then needed for these kind of integral (crust thickness) or mixed (efficiency) descriptors.

If the catalyst grain has a trivial shape (infinite plate and cylinder, sphere), simple analytical formulas are amenable. Yet, non-trivial grain shapes cannot be handled so easily and rises some questions on how to properly integrate such quantities. Commercial catalysts under internal diffusional limitations have indeed often non-trivial shapes. Callejas et al., by trying to evaluate the efficiency of HDM catalysts with a trilobe extruded shape, explicitly raise this lack of a proper method of integration (Callejas et al. 2001). Therefore there is a need for a general integration method able to cope with irregular object shapes. The purpose of this paper is precisely to fulfill this need. Fortunately, processes such as reaction-diffusion or imbibition lead to element distribution closely related to the distance to the surface of the object. This can be the start point for the design of a flexible method to extract global quantities from element distribution profiles. To illustrate our approach, we will focus on the 
computation of the global concentration of a grain from the concentration profile. It is justified by the fact that it is the simplest integral quantity and it can be compared to bulk analysis. The approach may obviously been adapted to compute other integral criterion such as crust thickness or mixed quantities such as catalyst efficiency.

This paper is organized as follow. In the first section, in the definition of the global concentration, we make a change in the integration variable introducing the distance to the border of the grain. The integration on the object is then performed through the computation of the cumulative histogram of the distance transform of the object. The methods for synthetic data generation and experimental profiles acquisition on an aged HDM catalyst are described. The second section presents the results showing the robustness and accuracy of the method and its applicability to a real case. In a third section we discuss the limits of this methodology and exploit the results from the real case example.

\section{Materials and Methods}

\section{$\underline{\text { Using the distance transform for integration }}$}

We will restrain the scope of the method to extruded objects from which an orthogonal section to the direction of extrusion completely defines the shape of the object. The generalization to true three dimensional shapes is straightforward, providing that the volume of the object is known instead of just one section. Imagine we want to compute a global quantity $I_{f}$ over the grain G defined by:

$$
I_{f}=\iiint_{\mathrm{G}} f(\vec{r}) d V
$$

Where $f(\vec{r})$ is a local quantity. Using this definition the global mass concentration $C$ of an element in a grain is equal to: 


$$
C=\frac{I_{\rho c}}{I_{\rho}}
$$

Where $c$ is the local mass concentration and $\rho$ the local density so that $I_{\rho c}$ is the total mass of element in the grain and $I_{\rho}$ is the mass of the grain. If we assume that the density $\rho$ is constant, the global concentration is equal to:

$$
C=\frac{I_{c}}{I_{\mathbb{1}}}
$$

Where $\mathbb{1}$ is the function equal to 1 everywhere in the grain (characteristic function) so that $I_{\mathbb{1}}$ is the volume $V$ of the grain.

We now make the assumption that $f$ does only depend on $y$, the distance from the surface of the grain. This assumption is true for trivial shapes (infinite plate, cylinder or the sphere) with an element distribution having the symmetry of the object. It is expected to be approximately fulfilled for element profiles generated by a physical mechanism where distance to the surface of the grain has an importance which is the case for reaction-diffusion or imbibition processes. Under this assumption, Equation (1) transforms to:

$$
I_{f}=\int_{0}^{y_{M A X}} f(y)\left|\frac{d V}{d y}\right| d y
$$

Very often, the local measure $f$ is only known in some discrete points $x_{j}$, typically on a line profile across the grain. The Figure 1 illustrates the general geometry of an extruded grain and the analyzed profile points $x_{j}$. Setting:

$$
\left\{\begin{aligned}
w(y) & =\left|\frac{d V}{d y}(y)\right| \\
f_{i} & =f\left(x_{i}\right)
\end{aligned}\right.
$$

Using the trapezoidal rule, we obtain a good approximation for $I_{f}$ : 


$$
2 I_{f} \approx \sum_{j=1}^{N_{T}-1}\left\{\left|y_{j+1}-y_{j}\right|\left[w_{j+1} f_{j+1}+w_{j} f_{j}\right]\right\}
$$

The evaluation of $I_{f}$ is then turned into the evaluation of functions $y(x)$ and $w(x)$. This can be easily done using the distance transform of a cross-section image of the extruded grain as illustrated in the Figure 2. The detailed workflow for the numerical evaluation of functions $y(x)$ and $w(x)$ is exposed in Appendix.

\section{$\underline{\text { Simulation of concentration maps }}$}

In order to validate the above methodology, we have simulated concentration maps using the simplest model for metal deposition under chemical reaction. Assuming that the precursor of the deposit does not plug the pores, that the diffusion of the precursor in the catalyst obeys a Fick diffusion behavior with effective coefficient $D_{e}$, an isothermal grain and that the deposit follows a first order kinetic of kinetic constant $k$, the steady state concentration of precursor obeys the following equation:

$$
D_{e} \nabla^{2} c-k c=0
$$

With Dirichlet condition limits at the border of the grain:

$$
c(\vec{r})=c_{0} \quad \forall \vec{r} \in \partial \mathrm{G}
$$

Where $\partial \mathrm{G}$ is the frontier of the grain G. Neglecting the transient deposit, the concentration $c_{d}$ of deposited metal is equal to:

$$
c_{d}=\frac{\varepsilon k t}{(1-\varepsilon) \rho} c
$$

Where $\varepsilon$ is the internal porosity and $\rho$ the structural density of the catalyst. Hence, the metal deposit profile is directly proportional to the steady state concentration profile of the precursor. The catalyst efficiency $\eta$ is defined as the rate of precursor conversion in the grain 
over the theoretical rate without diffusion limitations (Davis \& Davis, 2012). For a first order reaction:

$$
\eta=\frac{\int k c d V}{c_{0} k V}=\frac{\int c d V}{c_{0} V}=\frac{C}{c_{0}}
$$

Where $V$ is the volume of grain and $C$ is the global concentration in the grain. Under these simple assumptions, the global concentration $C$ is then equal to the catalyst efficiency $\eta$ for $c_{0}=1$

For trivial shapes (infinite plate and cylinder, sphere) the solutions of Equations 7 and 8 and the catalytic efficiency (hence the global concentration $C$ in the grain) are well known (Davis \& Davis, 2012). The solutions for an infinite cylinder, summarized in Table 1, are parameterized by the Thiele modulus $\phi$ which gives the balance between reaction and diffusion characteristic times in the grain. High Thiele modulus corresponds to the diffusion limited regime and hollow profiles whereas low Thiele modulus corresponds to the chemical regime and flat profiles. For irregular grain shapes such as the tetralobed shape of Figure 2, Equations 7 and 8 are solved by finite element method using Comsol Multiphysics v4.3a with a 5000 elements mesh. Integration of the concentration map gives the global concentration in the grain equal to the catalyst efficiency $\eta$ for $c_{0}=1$.

Synthetic profiles are extracted from the simulated concentration map and processed to obtain global concentration $C$ using the distance transform method described previously. This global concentration is compared with the theoretical one $\eta$ predicted by the models (from Table 1 or by Comsol Multiphysics). We define the relative error $\varepsilon_{C}$ as:

$$
\varepsilon_{C}=\frac{|C-\eta|}{\eta}
$$


This accuracy does only take into account the sampling of points, it does not consider the uncertainties due to counting statistics of X-ray intensities. The profiles are generated using either a constant step or a variable one. Constant step is simply calculated dividing the profile length by the number of acquisition points minus one. This constant step is not well suited for very hollow profiles where the elements are absent in the core of the grain and hence where most points of the profile have a measured concentration below the detection limit. For hollow profiles, it is therefore more efficient to bring closer the point in the vicinity of the grain surface and consequently move away those in the core of the grain. For hollow profiles, we propose to construct a profile of $N$ points by three successive segments of fixed step, a first segment in the crust, a second in the core and the last one in the opposite crust. Step length of each segment is computed following these rules:

- The number of points in each segment is set to $\frac{N}{3}$.

- The length of the crust is the distance to the grain surface where the concentration falls below one tenth of its maximum (at the surface). If this point does not exist or if the calculated step length in the crust is larger than in the core, then a fixed step profile suits.

- The step must not be lower than $5 \mu \mathrm{m}$, the order of magnitude of the lateral resolution for alumina catalysts with porosity being around to 0.75 at $20 \mathrm{kV}$.

This procedure is not optimal for accuracy but is sufficiently simple to be applicable for real measurements. The positions of the analysis points are easily calculated from the start and end points of the profile, the total number of points and the crust thickness. This crust thickness may be evaluated by a previous qualitative intensity profile.

\section{$\underline{\text { EPMA and XRF measurements on an aged catalyst }}$}


A HDM catalyst in tetralobed shape was submitted to a $3000 \mathrm{~h}$ long stability test in a fixed bed reactor. The reactor was unloaded and two sampling made from inlet and outlet of the reactor. Samples were washed by toluene and calcined under air to remove remaining organic species and coke. This step also transforms the metal sulphides phases into oxides (Toulhoat et al., 1990). Catalysts grains were embedded in an epoxy resin (Struers Specifix-40) which was prepolymerized to prevent from carbon contamination (Sorbier et al., 2004) and polished to obtain a flat section (see Figure 2 (a)). A thin conductive carbon layer was deposited to prevent surface charging effects. Samples were analyzed using a JEOL JXA8100 electron microprobe with a $20 \mathrm{keV}$ and $100 \mathrm{nA}$ electron beam. Other measurement parameters are reported in Table 2. Oxygen is calculated by stoichiometry assuming the oxide formula of Table 2. Standards are pure metals for $\mathrm{Ni}, \mathrm{V}$ and $\mathrm{Mo}$ and sapphire $\left(\mathrm{Al}_{2} \mathrm{O}_{3}\right)$ for $\mathrm{Al}$, all covered by a thin carbon layer. For each sample, five profiles are measured on five different catalyst grains along the small and long axis of the tetralobed shape. Each profile is acquired with 60 points resulting in a constant step of about $22 \mu \mathrm{m}$ along the short axis and $32 \mu \mathrm{m}$ along the long axis. The first and the last points lay in the embedding resin. Local measured concentrations are then processed using the method of the distance transform described above to obtain the global concentration in the grain. For XRF analysis, calcined extrudates from inlet were crushed in a mortar, diluted about 50 times in a lithium, borate and phosphate glass and molded around $1000{ }^{\circ} \mathrm{C}$ to form glass pearls. Pearls were cooled and analyzed using a wavelength-dispersive X-ray Fluorescence (PW2404, PANalytical). The quantitative analysis was performed using calibration curves obtained from reference pearls containing weighted amount of elements. Sampling from reactor outlet was insufficient to perform XRF as in this reactor region, the HDM catalyst was mixed with a different finishing catalyst and sorting of extrudates had to be done by hand.

\section{Results}


Parametric study on infinite cylinder: number of points and variable step

To study the effect of the number of points and the variable step strategy, we have simulated concentration maps for an infinitely long cylinder of $0.9 \mathrm{~mm}$ diameter with $0.5 \mu \mathrm{m}$ pixel size for different Thiele modulus ranging from 0.01 to 100 (analytical formula of Table 1). The profiles have been taken along the diameter of the cylinder. The Figure 3 presents the accuracy of the global concentration $C$ in the grain according to the Thiele modulus value for synthetic constant step profiles. As expected, the error is increasing when the profile becomes less flat (when $\phi$ increases) and when the number of points used to describe the profile decreases. Using a 60 points constant step profile yields to an error lower than $1 \%$ for $\phi<$ 10. For hollow profiles it is more efficient not to sample evenly the analysis points. The Figure 4 shows the errors for a 60 points profile along a $0.9 \mathrm{~mm}$ diameter infinite cylinder for both fixed and variable step samplings as described in previous section. For $\phi<3$ the profiles are too flat to imply a variable step sampling by our proposed method. For $\phi>3$, variable step sampling is effectively performed and a significant lowering of the error is observed.

For the tetralobed shape, we have simulated concentration maps of a $2.07 \mathrm{~mm}$ (long axis) infinitely long tetralobe by finite element method with Comsol Multiphysics. Concentrations maps with a $1.4 \mu \mathrm{m}$ pixel size were generated from Comsol Multiphysics. Examples of such concentration maps are shown in Figure 5. As the tetralobed shape has two different and nonequivalent symmetry axis, we have generated synthetic profiles of 60 points using variable step. The two different symmetry axis (called respectively short and long in the following) are imaged Figure 6. These two axis both contains the center of the tetralobe where the distance to the surface is maximized. Hence, both axis explore all the possible distances from the surface. Figure 7 compares the global concentration $C$ for $c_{0}=1$ integrated along the two axis and assuming either a tetralobe or a cylinder of diameter equal to the length of the profile 
with the global concentration computed from the full map. We can notice that with the tetralobe model, the integration along the short axis always underestimates $C$ whereas integration along the long axis overestimates it. This suggests that combining short and long axis should give a more accurate estimator. This combination can be done by two different ways: either by taking the arithmetic mean of the short and long axis estimators or by integrating along the bisecting line between short and long axis called in the following, the median axis. For the cylinder shape hypothesis, no such overestimation along the long axis and underestimation along short axis are noticed in the full Thiele modulus range. Figure 8 shows the associated errors for the integration along short, long and median axis and the two shape hypothesis (cylinder or tetralobe). As expected, for low Thiele modulus (flat profiles), all the integration strategies give low errors.

The Figure 9 and 10 show the mean distribution of deposited vanadium and nickel in the catalyst grain for samples from respectively, the inlet and the outlet of the reactor. We clearly observe an uneven distribution for both metals. The extracted mean global concentrations and standard deviations for the five measured grains are reported in Table 3 using the average of short and long axis. We clearly observe a decreasing of the trapped metal concentration from inlet to outlet of the reactor.

\section{Discussion}

We have proposed a general integration method of local measurement for global quantities at the grain scale. The same formalism is applicable to trivial shapes (infinite plate and cylinder, sphere) for which analytical formulas are given. The methodology has been illustrated for extruded shape where only a high resolution picture of the grain section is needed. The extension to full three-dimensional shapes requires a measure of the whole volume of the object (by tomographic techniques for example). This clearly restrains its simple (routine) 
application to extruded forms with lengths larger than inscribed diameter. The methodology should also fail for very irregular object surface such like fractal. The smoothing of the $\frac{d n}{d y}(y)$ and $y(x)$ functions implies a rather regular behavior of these functions except in some points away from the surface. Using unsmoothed numerical expression for $\frac{d n}{d y}(y)$ is dangerous since exact distance calculation of small distances is very inaccurate due to poor sampling.

The application of the method on synthetic data allowed us to evaluate the sampling errors in function of the number of points and flatness of the profile for an application in chemical engineering. For the infinite cylinder, increasing the number of points has a direct influence on accuracy: for rather flat profiles $(\phi<1)$ : doubling the number of points divides the error by about 5 (a factor 4 is expected for a trapezoidal rule). The increasing of sampling points has however two limits. The first one is the linear increase of analysis time with the number of points. The second is that for a sufficiently large number of points and small object, the step length may become smaller than the lateral resolution of the local measurement. Lowering the step length below the lateral resolution is highly questionable as local analysis characterization techniques (EPMA for example) assume the homogeneity of composition of the sample at lateral resolution scale. Handling continuous lateral composition gradients in the interaction volume is difficult even with Monte-Carlo simulations. It is also worth noting that Thiele modulus higher than 10 gives very shallow profiles that may not be resolved by EPMA. Fortunately, such profiles should be rarely observed on catalyst grains as they suppose a very low catalyst efficiency (lower than 10\%). Usually, the textural properties of the catalyst support is adapted in order to avoid such low efficiencies. The variable step sampling method allows a further reduction of the sampling error without increasing the analysis time. For moderate values of Thiele modulus $(\phi<10)$ and 60 points, the variable step yield a sampling error lower than $0.6 \%$. 
For shapes with lower symmetries than the cylinder, the question of the choice of the integration axis rises. Obviously, the integration axis must explore all the distances to the surface of the grain. For the tetralobe, integration along short or long axis gives important relative errors up to $18 \%$. The overestimating of $C$ along long axis and underestimating along short axis can be deduced from the simulated maps. The Figure 5 for $\phi=1$ clearly shows steep concentration gradients in the hollows (concave parts, short axis) and much milder gradients near the lobes (convex parts, long axis), which causes an underestimation if the hollows are sampled and an overestimation if the lobes are sampled. This difference also demonstrates that the hypothesis telling the local concentration depends only on the distance to the surface of the grain is not fulfilled. Taking into account this constant over or underestimation can be exploited to produce more accurate estimators either by a simple averaging or by the integration trough the median line (Figure $6(\mathrm{c})$ ). Both strategies yield relative errors lower than 5\% as shown in Figure 8. However, these strategies have serious drawbacks. Making the average between short and long axis doubles the analysis time and moderately complicates data processing. Integration along median axis is practically difficult. If short and long axis are easy to detect on real sample pictures, it is not the case for the median axis. Even if this solution seems interesting since it neither lengthens the analysis time nor complicates data processing, it is not practically applicable due to the difficulty of defining the start and end points of the median profile for a real application.

The only proposed strategy in the literature is to perform the integration assuming a cylinder shape with diameter equal to the length of the profile. Using this assumption, quite good estimators are obtained for $\phi \leq 2$ along the long axis and $\phi \geq 6$ along the short axis. It is however difficult to recommend this strategy since it gives large errors for an important range of Thiele modulus $(2<\phi<6)$ which is the transition range between chemical and diffusion regime where the shape of the grain slightly impacts the catalyst efficiency. With this 
considerations, our method of integration using the distance transform yields much better estimates whatever the hollowness of the profile.

These above conclusions are difficult to generalize to any extruded form (trilobe, other tetralobed shapes, ...) or other global quantities than global concentration. It is however expected that averaging the measures on each non-equivalent symmetry axis should provide the best estimator of any global quantity for any grain shape. It is also difficult to extrapolate errors for other global quantities not proportional to $C$, even keeping the same tetralobed shape.

Measurements on the aged catalyst (Figures 9 and 10) reveals uneven deposited metal distributions that are attributed to internal diffusional limitations of the deposition process. These moderately hollowed profiles suggest a moderate Thiele modulus around 1 and hence a rather good catalytic efficiency around $80 \%$. As the catalyst has a significant macroporosity, its polished surface has local holes that creates roughness and local loss or increasing of X-ray emission compared to a flat sample (Gauvin\&Lifshin, 2000). This explains the important local fluctuations of concentrations, far larger than expected from counting statistics (total Xray count are around $4 \times 10^{4}$ for $\mathrm{Ni}$ and $10^{5}$ for $\mathrm{V}$ ). The overall effect of this roughness is a global signal loss, leading to sum of concentration in the range of $90 \%$ for both samples (Sorbier et al., 2001). However, since this macroporosity is, on average, uniformly distributed within the grain, the global shape of the element distribution profile is not modified. The relative accuracy between global concentration obtained by integration from element profiles and by XRF is within the order of magnitude expected, $10 \%$ for vanadium and $2 \%$ for nickel. It validates the methodology of integration by the distance transform, showing that it is possible to extract quite accurately global quantities from element profiles. 
Comparison of the mean deposited concentration between inlet and outlet of the reactor shows that metal removal proceeds progressively along the reactor as already observed (Tamm et al., 1981);(Agrawal\&Wei, 1984). We can conclude that the trapping is not complete (since metal is significantly deposited in the outlet) and that catalyst in the outlet has not reached its maximum trapping concentration (less metal in the outlet than in the inlet). A comparison between vanadium and nickel profiles gives valuable information on the relative reactivity of the two metals precursors. Mean deposited concentration of nickel decreases less along the reactor than vanadium. Besides, the deposited vanadium profiles are more concave (higher Thiele modulus) than nickel profiles suggesting a better nickel trapping efficiency in the catalyst grain as others already noticed (Tamm et al., 1981);(Callejas et al., 2001);(Marafi et al.,2007). To rephrase, nickel trapping is more efficient at the grain scale but less efficient at the reactor scale. To compare the relative reactivity or transport of both metals, a model at the reactor scale must be taken into account.

\section{Conclusion}

We have proposed an original method to integrate local measurements for complex grain shapes using the distance transform. We exemplified this methodology in the field of chemical engineering where global concentrations in the catalyst grain have to be calculated from element distribution profiles in the grain. Synthetic profiles extracted from a simple deposition model allowed us to test the accuracy of the method. On cylinders with a sufficiently high number of points (60), a variable step sampling strategy and well defined profiles, the methodology yield relative errors below $1 \%$ for well resolved profiles. For a less regular shape such as a tetralobe, estimators of global concentration are much more biased. The best estimator is obtained by an averaging of the integrals along the two non-equivalent symmetry axis of the object, yielding a relative error lower than 5\%. We have thus shown that 
the global concentration inside a catalyst grain can be rather accurately extracted from an elemental profile even for complex grain shapes.

To demonstrate the practical applicability of the method, we have applied the proposed methodology on a real case. An aged HDM catalyst has been sampled in the inlet and the outlet of the reactor and analyzed by EPMA. Concentration profiles let us conclude that the trapping of nickel is more efficient than vanadium at the grain scale whereas the contrary is observed at the reactor scale. Integrated global concentration are found close to XRF measurements, confirming the validity of our methodology of integration on complex shapes.

\section{Appendix: Calculation of weighting function}

Global quantities can be obtained from evaluation of functions $y(x)$ and $w(x)=\left|\frac{d V}{d y}(x)\right|$ (Equation 6). It is more convenient to normalize the function $\frac{d V}{d y}$ by the volume $V$ of the grain:

$$
\frac{d n}{d y}=\frac{1}{V} \frac{d V}{d y}
$$

For trivial shapes, the functions $y(x)$ and $\frac{d n}{d y}(x)$ are analytical and are reported in Table 4 . For less symmetrical shapes, these functions may be easily evaluated using the distance transform of an image of the grain and the position of the line profile. Let $G$ be the set of pixels belonging to the grain and $G^{c}$ its complement. The distance transform of $G, G^{D T}$ is defined by (Rosenfeld and Pfaltz, 1966):

$$
\mathrm{G}^{\mathrm{DT}}=\left\{y(x)=\min _{a \in \mathrm{G}^{\mathrm{c}}}\|x-a\|, x \in \mathrm{G}\right\}
$$

This transformation is well known and used in the image processing community (Fabbri et al., 2008). The cumulative histogram $n(y)$ of fraction of pixels of $\mathrm{G}^{\mathrm{DT}}$ having a value lower than $y$ is then computed. Numerical differentiation $n(y)$ using finite difference scheme is performed: 


$$
\left.\frac{d n(y)}{d y}\right|_{y=y_{i}}=\frac{n\left(y_{i+1}\right)-n\left(y_{i-1}\right)}{y_{i+1}-y_{i-1}}
$$

As numerical computation of the distance transform and numerical derivation introduce numerical noise, it is necessary to smooth $\frac{d n}{d y}(y)$ function. The $\frac{d n}{d y}(y)$ function is thus leastsquare fitted with square root weighting by a line for $y \in\left[0, y_{1}\right]$ and by four sixth order polynomials for $y \in\left[y_{1}, y_{\mathrm{MAX}}\right]$. The value $y_{1}$ is obtained by an extensive search of the minimum of the obtained $\chi^{2}$ for $y_{1} \in\left[2 \Delta y, y_{\mathrm{MAX}}-4 \Delta y\right]$ with $\Delta y$ corresponding to 10 pixels distance. The function $y(x)$ is obtained directly from the position of the line profile and the distance transform. It is also smoothed by piecewise polynomials. The maximum of $y(x)$, $y_{\mathrm{MAX}}=y\left(x_{2}\right)$ is found. The function $y(x)$ is least-square fitted using square root weighting by fourth order polynomials, two for $x \in\left[0, x_{1}\right]$, three for $x \in\left[x_{1}, x_{2}\right]$, three for $x \in\left[x_{2}, x_{3}\right]$ and two for $x \in\left[x_{3}, x_{\text {MAX }}\right]$. The optimum values $x_{1}$ and $x_{3}$ that minimize the obtained $\chi^{2}$ are extensively searched for $x_{1} \in\left[3 \Delta x, x_{2}-4 \Delta x\right]$ and $x_{3} \in\left[x_{2}+4 \Delta x, x_{\mathrm{MAX}}-3 \Delta x\right]$ where $\Delta x$ corresponds to 10 pixels. This strategy supposes that a high resolution image of the grain section is available, typically to obtain distance and profile length of at least few hundreds of pixels.

As an illustration of the methodology, Figure 2 summarizes the workflow. A cross section of the extruded grain is acquired using any suitable imaging technique: Scanning Electron Microscopy (SEM) in backscattered electrons mode, optical microscopy, .... The image of the section is segmented and the distance transform of the segmented image is computed. The distance transform now contains in its pixel the distance to the surface of the grain. The fraction of the points $n(y)$ of the Figure 2 (c) having a value lower than $y$ is plotted on Figure 11. The first derivative of $n(y)$ is plotted on Figure 12 superimposed with least-square fitted piecewise polynomials. The function $y(x)$ for the profile defined in Figure $2(d)$ is plotted on Figure 13, superimposed with fitted piecewise polynomial. 
It is worth noting that the value of $\frac{d n}{d y}(y)$ at $y=0$ gives a robust measure of the surface $S$ to volume $V$ ratio of the grain which is a key parameter to evaluate catalyst efficiency under internal diffusion limitation (Bischoff, 1965):

$$
\left.\frac{d n}{d y}\right|_{y=0}=y_{M A X} \frac{S}{V}
$$




\section{References}

Agrawal, R. \& WeI, J. (1984). Hydrodemetallation of Nickel and Vanadium Porphyrins. 2. Intraparticle Diffusion. Ind. Eng. Chem. Process Des. Dev. 23 515-522.

BISCHOFF, K. (1965). Effectiveness factors for general reaction rate forms. AIChE Journal 11 $351-355$.

Callejas, M., Martinez, M., Fierro, J. Rial, C. Jimenez-Mateos, J. \& Gomez-Garcia F. (2001). Structural and Morphological Study of Metal Deposition on an Aged Hydrotreating Catalyst. Appl. Catal. A 220 93-104.

DAVIS, M. \& DAVIS, R. (2012). Fundamentals of Chemical Reaction Engineering. New York: Courier Dover Publications.

Fabbri, R., Costa L., Torelli J. \& Bruno, O. (2008). 2D Euclidean distance transform algorithms: A comparative survey. ACM Comput. Surv. 40 1-44.

Furimsky, E. \& MASsoth, F. (1999). Deactivation of Hydroprocessing Catalysts. Catal. Today 52 381-495.

GAuvin, R. \& LifShin, E. (2000). Simulation of X-Ray Emission from Rough Surfaces. Mikrochim. Acta 132 201-204.

Marafi, A., Hauser, A. \& Stanislaus, A. (2007). Deactivation Patterns of $\mathrm{Mo} / \mathrm{Al}_{2} \mathrm{O}_{3}$, $\mathrm{Ni}-\mathrm{Mo} / \mathrm{Al}_{2} \mathrm{O}_{3}$ and $\mathrm{Ni}-\mathrm{MoP} / \mathrm{Al}_{2} \mathrm{O}_{3}$ Catalysts in Atmospheric Residue Hydrodesulphurization. Catal. Today 125 192-202.

Merdrignac, I., Roy-Auberger, M., Guillaume, D. \& Verstraete, J. (2013). Hydroprocessing and Hydroconversion of Residue Fractions. In Catalysis by Transition Metal Sulphides, Toulhoat, H. \& Raybaud, P. (Eds.), pp 679-737. Paris: Editions Technip. 
Rosenfeld, A. \& Pfaltz, J. (1966). Sequential operations in digital picture processing. JACM 13 471-494.

Sorbier, L., Rosenberg, E. \& Merlet, C. (2001). Monte Carlo Simulations of Rough and Porous Alumina. In Advanced Monte Carlo for radiation physics, particle transport simulation and applications, Kling, A., Barao, F., Nakagawa, M., Tavora, L. \& Vaz, P (Eds), pp. 389-394. Berlin: Springer-Verlag.

Sorbier, L., Rosenberg, E. \& Merlet, C. (2004). Microanalysis of Porous Materials. Microsc. Microanal. 10 745-752.

Sorbier, L., Gay, A.S., FÉCAnt, A., Moreaud, M. \& Brodusch, N. (2012). Measurement of palladium crust thickness on catalyst by EPMA. IOP Conference Series: Materials Science and Engineering 32012023.

SORBIER, L. (2013). Determining the Distribution of Metals by Electron Probe Micro Analysis. In Catalysis by Transition Metal Sulphides, Toulhoat, H. \& Raybaud, P. (Eds.), pp 407-411. Paris: Editions Technip.

TAmm, P., Harnsberger, H. \& Bridge, A. (1981). Effects of Feed Metals on Catalyst Aging in hydroprocessing Residuum. Ind. Eng. Chem. Process Des. Dev. 20 262-273.

Toulhoat, H., Szymanski, R. \& Plumail, J.C. (1990). Interrelations between Initial Pore Structure, Morphology and Distribution of Accumulated Deposits, and Lifetimes of Hydrodemetallisation Catalysts. Catal. Today 7 531-568. 


\section{Tables}

Table 1: Thiele modulus $\phi$, steady-state concentration profile $c(r)$ and catalyst efficiency $\eta$ for an infinite cylinder of radius $R . I_{0}$ and $I_{1}$ are the modified Bessel functions of 0 and 1 order and $r$ is the distance to the axis of the cylinder.

Thiele modulus $\phi \quad$ Profile $\frac{c(r)}{c_{0}} \quad$ Efficiency $\eta$

$$
\frac{R}{2} \sqrt{\frac{k}{D_{e}}} \quad \frac{I_{0}\left(2 \phi \frac{r}{R}\right)}{I_{0}(2 \phi)} \quad \frac{I_{1}(2 \phi)}{\phi I_{0}(2 \phi)}
$$

Table 2: EPMA analysis parameters. $T$ is the peak and background acquisition times.

\begin{tabular}{ccccc}
\hline Element & Oxide & Line & $T(\mathrm{~s})$ & Crystal \\
\hline $\mathrm{Al}$ & $\mathrm{Al}_{2} \mathrm{O}_{3}$ & $\mathrm{~K} \alpha(2)$ & 20 & TAP \\
$\mathrm{Mo}$ & $\mathrm{MoO}_{3}$ & $\mathrm{~L} \alpha$ & 10 & PETJ \\
$\mathrm{V}$ & $\mathrm{V}_{2} \mathrm{O}_{5}$ & $\mathrm{~K} \alpha$ & 20 & LIFH \\
$\mathrm{Ni}$ & $\mathrm{NiO}$ & $\mathrm{K} \alpha$ & 10 & LIFH \\
\hline
\end{tabular}

Table 3: Mean global concentrations $C$ (wt \%) of deposited metals obtained from XRF and integration of EPMA profiles. Uncertainties are the standard deviations computed from the five profiles.

\begin{tabular}{cccc}
\hline \multirow{2}{*}{ Element } & \multicolumn{2}{c}{ Inlet } & Outlet \\
& XRF & EPMA & EPMA \\
\hline $\mathrm{V}_{2} \mathrm{O}_{5}$ & 33.9 & $37.5 \pm 4.1$ & $19.6 \pm 3.1$ \\
$\mathrm{NiO}$ & 4.80 & $4.90 \pm 0.32$ & $3.54 \pm 0.38$ \\
\hline
\end{tabular}


Table 4: $y(x)$ and $\frac{d n}{d y}(y)$ functions for trivial grain shapes. The line profile is taken along the grain diameter.

\begin{tabular}{lcc}
\hline Grain shape & $y(x)$ & $\frac{d n}{d y}(y)$ \\
\hline Infinite plate (thickness $2 R)$ & $\min (x, 2 R-x)$ & $\frac{1}{R}$ \\
Infinite cylinder (radius $R)$ & $\min (x, 2 R-x)$ & $\frac{2}{R}\left(1-\frac{y}{R}\right)$ \\
Sphere (radius $R)$ & $\min (x, 2 R-x)$ & $\frac{3}{R}\left(1-\frac{y}{R}\right)^{2}$ \\
\hline
\end{tabular}

\section{Figure legends}

Figure 1: General geometry for an extruded grain. An orthogonal section of the grain completely defines its volume. Microanalysis profile is performed on finite and discrete points $x_{j} . y$ is the distance to the surface of the grain.

Figure 2: General methodology for integration using the distance transform. (a) Scanning electron microscopy image of a polished section of the grain in backscattered electrons mode (b) segmented image (c) distance transform of segmented image in gray level (d) example of microanalysis points profile, in red, superimposed on the distance transform.

Figure 3: Accuracy of $C$ in function of Thiele modulus $\phi$ for fixed step profiles along the diameter of a cylinder. 
Figure 4: Accuracy of $C$ in function of Thiele modulus $\phi$ for fixed or variable step profiles along the diameter of a cylinder. The number of points is 60 .

Figure 5: Example of some concentration maps obtained by finite element method on the tetralobed shape with different Thiele modulus $\phi$. Concentration values vary from 0 (dark blue) to 1 (red at the surface of the grain).

Figure 6: The two symmetry axis of the tetralobed shape (a) short axis (b) long axis and (c) the median axis, the bisecting line between short and long axis.

Figure 7: Global concentration for $c_{0}=1$ in function of Thiele modulus $\phi$ for the tetralobed shape. Comparison of values computed from full concentration map and integrated from 60 point variable step profiles.

Figure 8: Accuracy of $C$ in function of Thiele modulus $\phi$ for the tetralobed shape. Integration is performed along a 60 points profile with variable step.

Figure 9: Average EPMA profiles of $\mathrm{Ni}$ and $\mathrm{V}$ along the short axis of tetralobes from the inlet of reactor. 
Figure 10: Average EPMA profiles of $\mathrm{Ni}$ and $\mathrm{V}$ along the short axis of tetralobes from the outlet of reactor.

Figure 11: Fraction of pixel $n(y)$ of Figure 2 (c) having a value lower than $y$.

Figure 12: First derivative of $n(y)$ computed from Figure 3 (symbols) and fitting by piecewise polynomials (line).

Figure 13: Distance to the surface of the grain $y(x)$ in function of the position along the profile for the profile defined in Figure 2 (d) (symbols) and fitting by piecewise polynomials (line). 
Figures

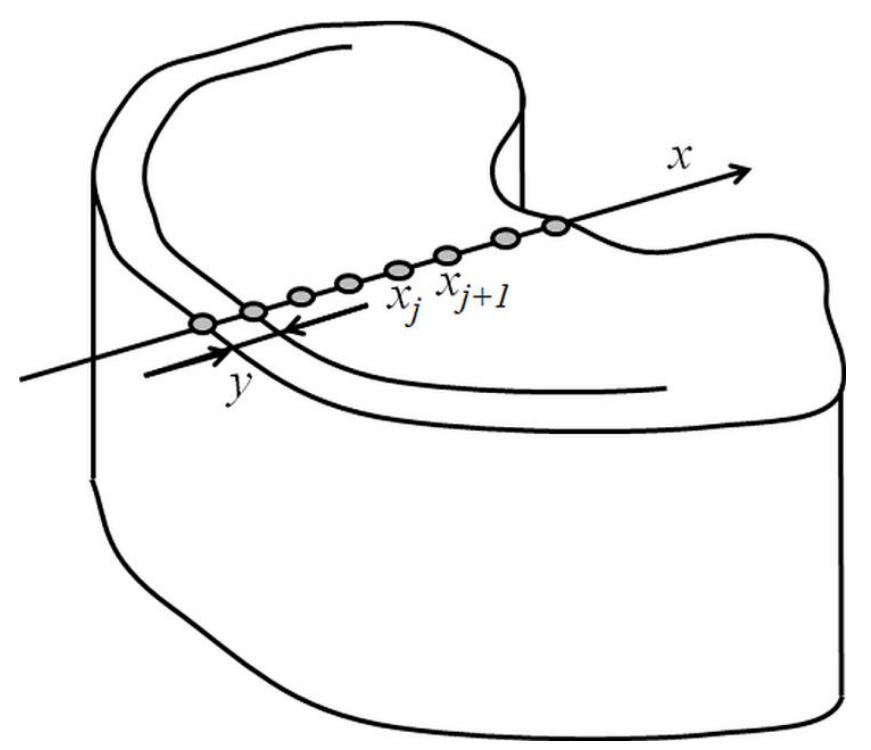

Figure 1

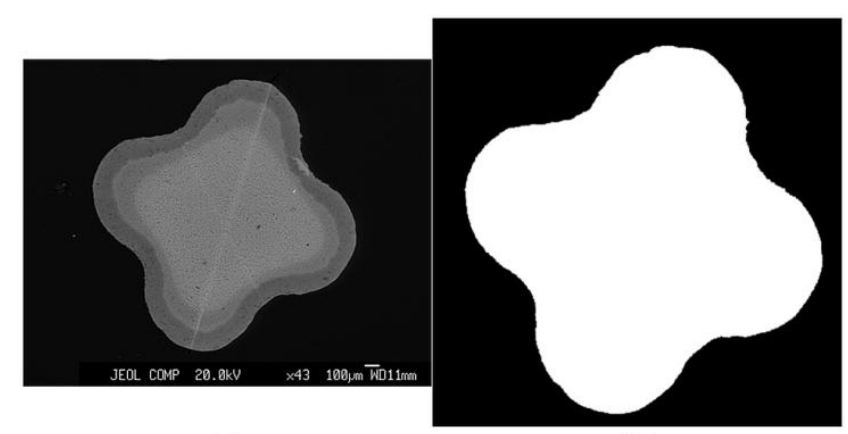

(a)

(b)

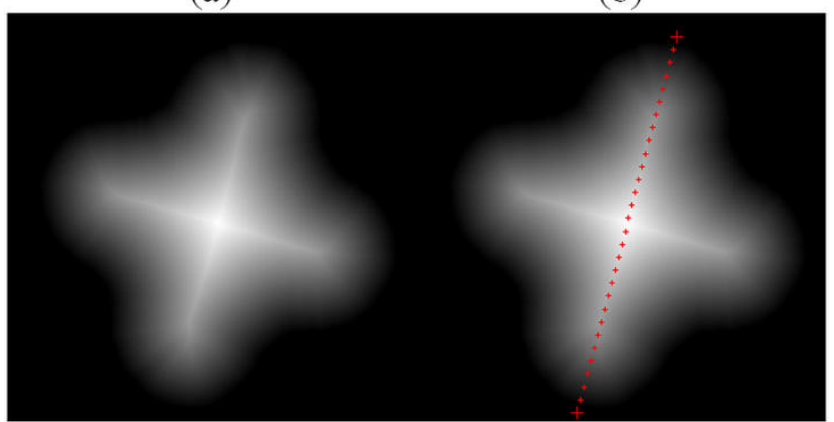

(c)

(d)

Figure 2 


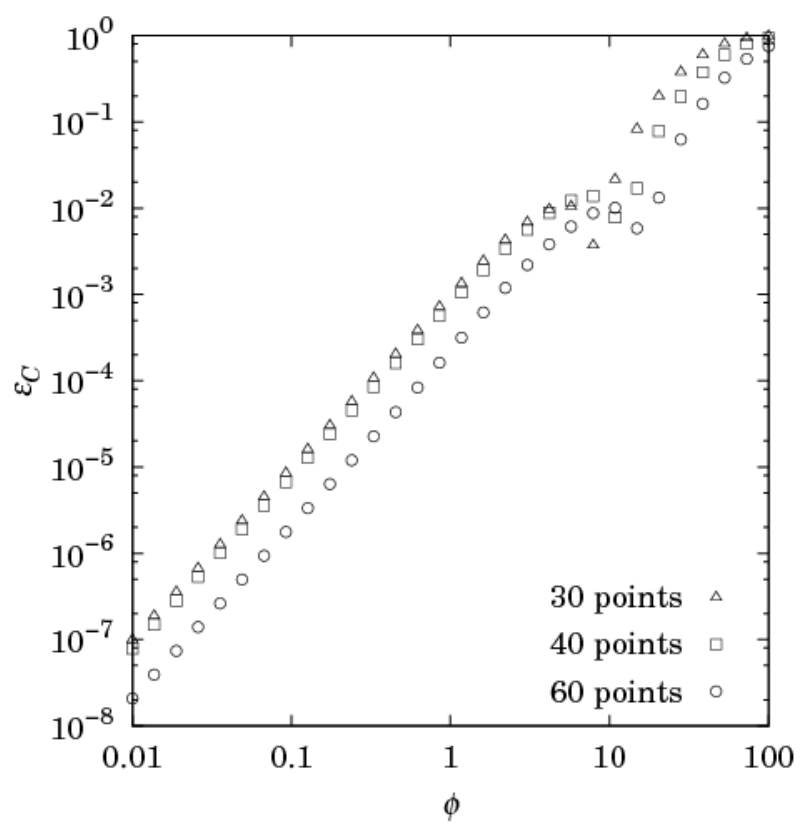

Figure 3

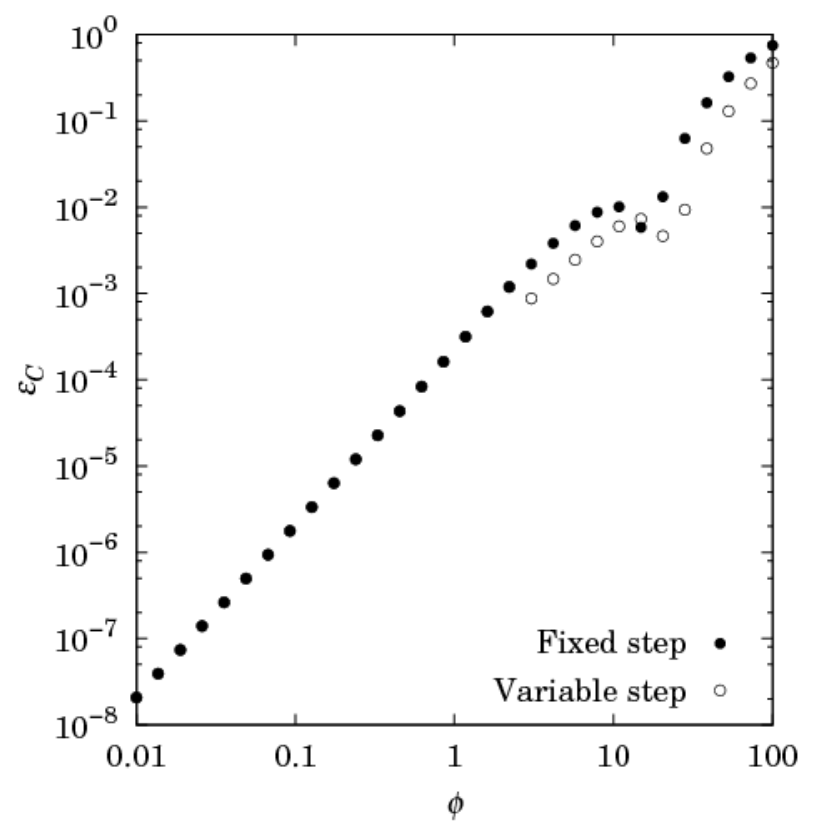

Figure 4 


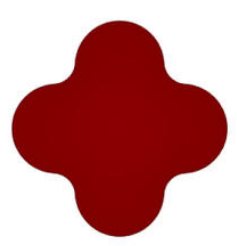

0.1

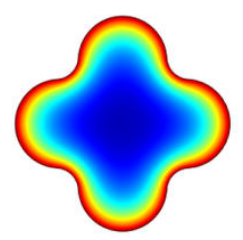

2.15

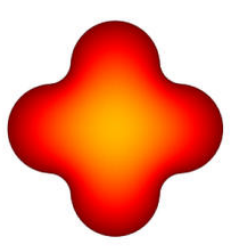

0.599

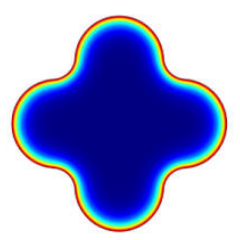

5.99
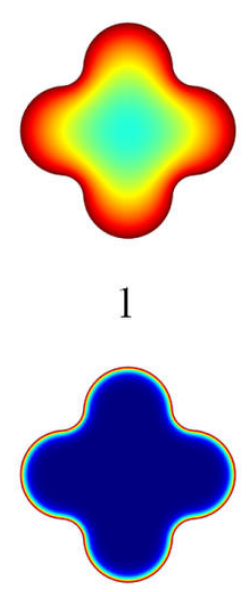

10

Figure 5

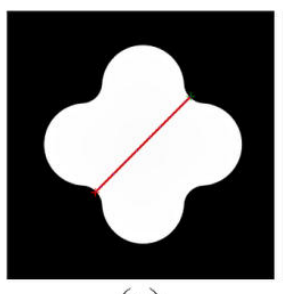

(a)

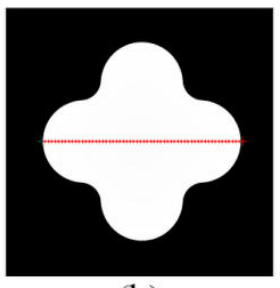

(b)

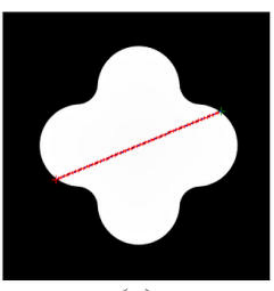

(c)

Figure 6 


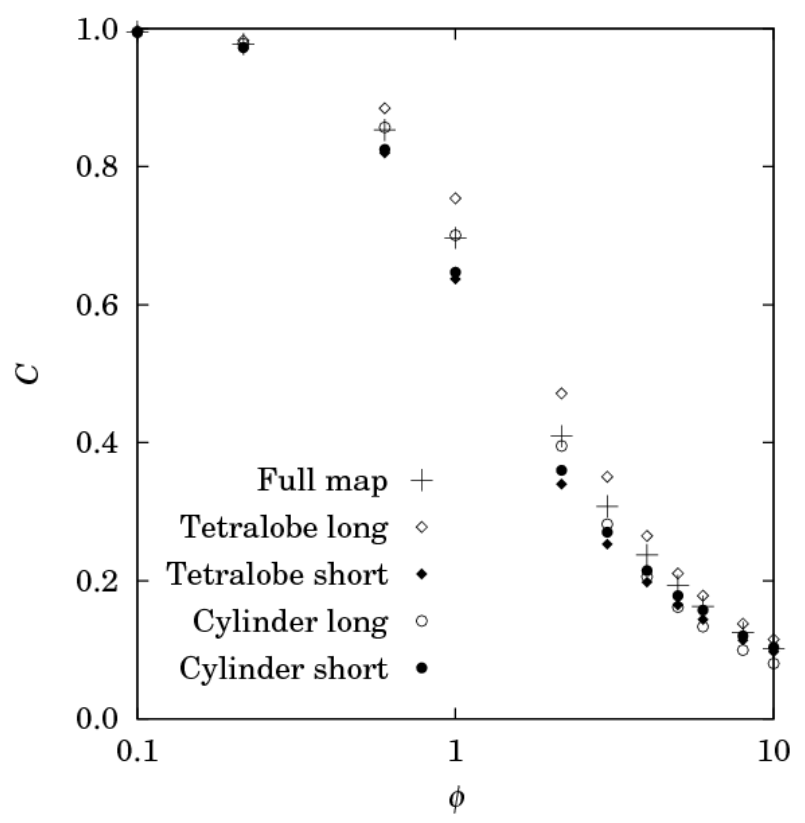

Figure 7

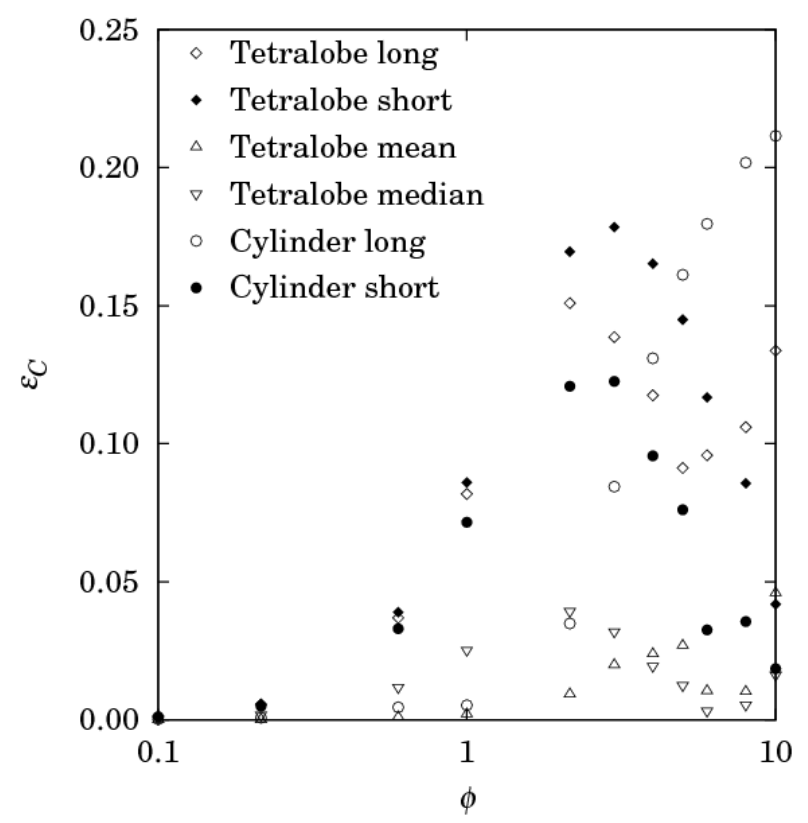

Figure 8 


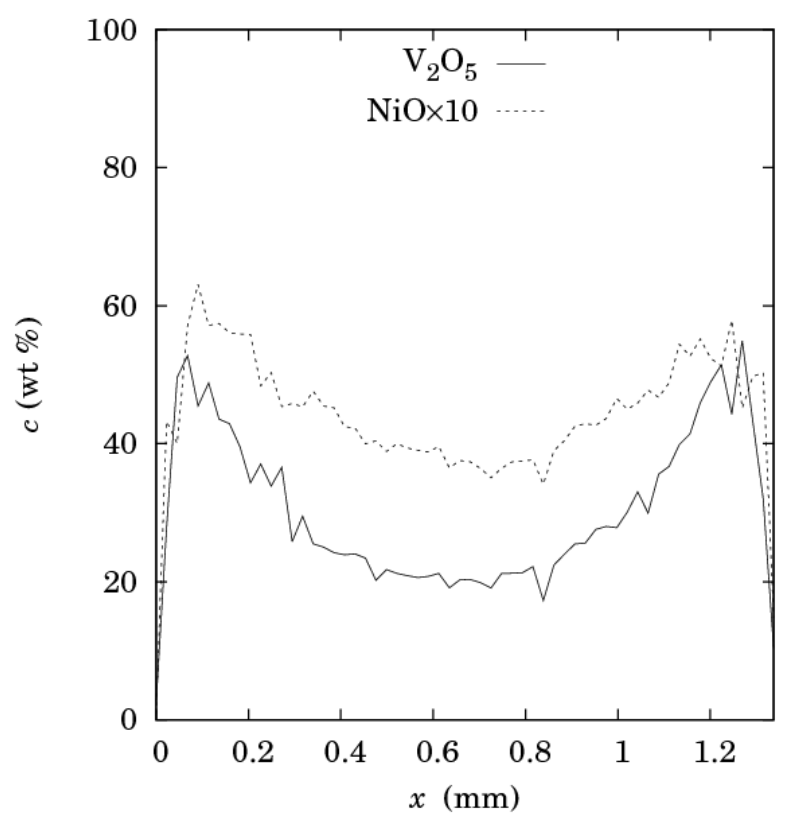

Figure 9

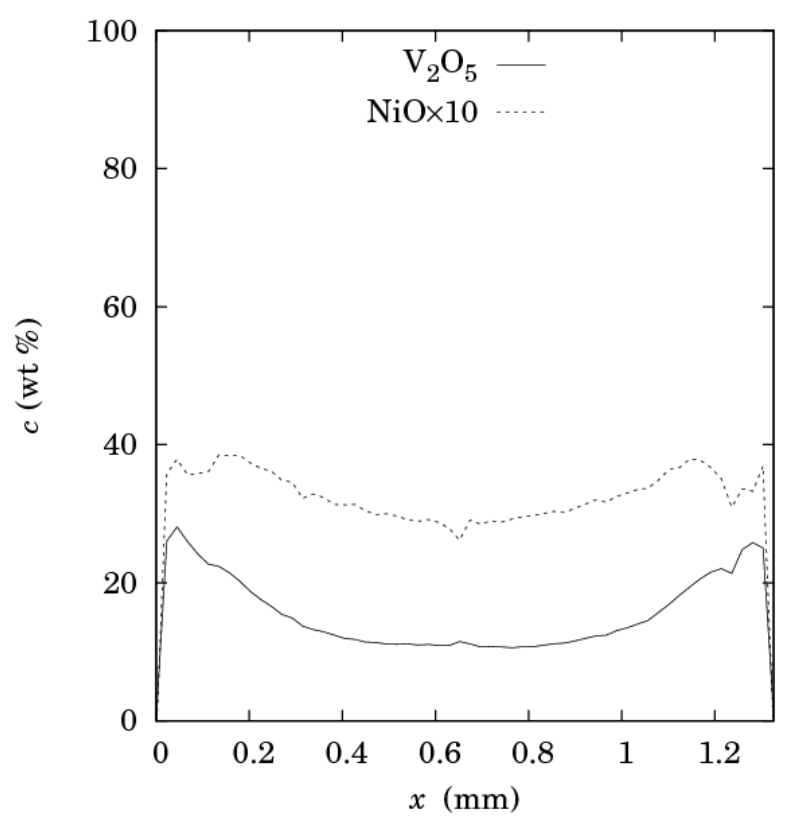

Figure 10 


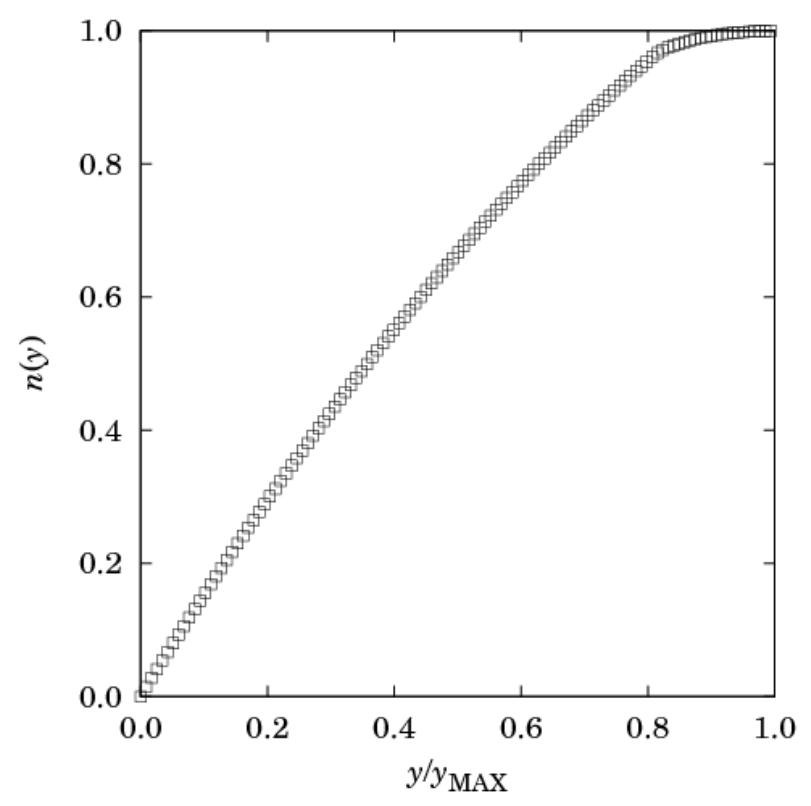

Figure 11

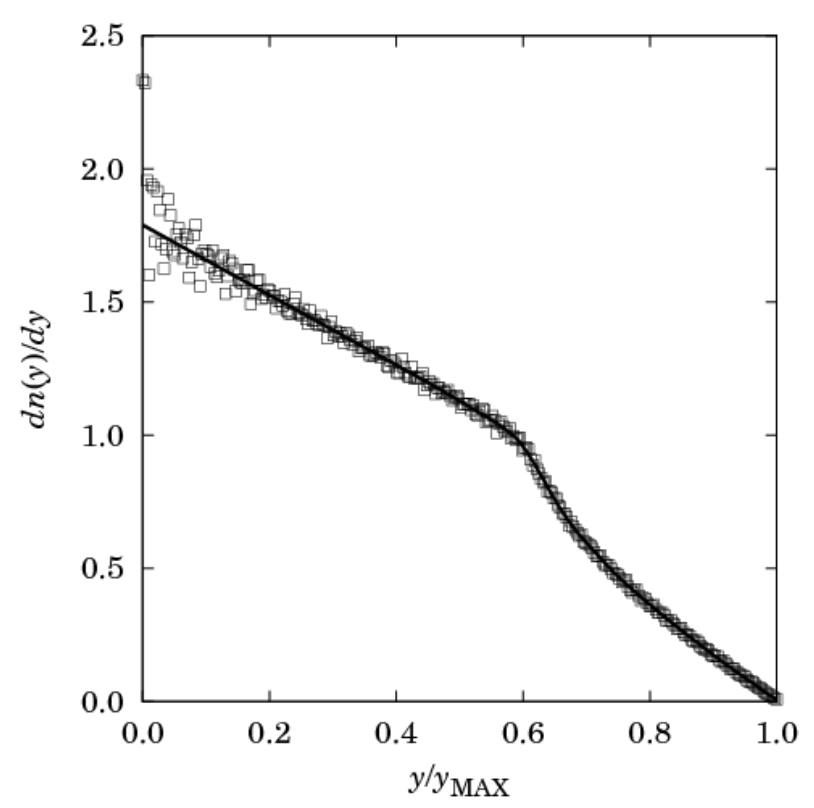

Figure 12 


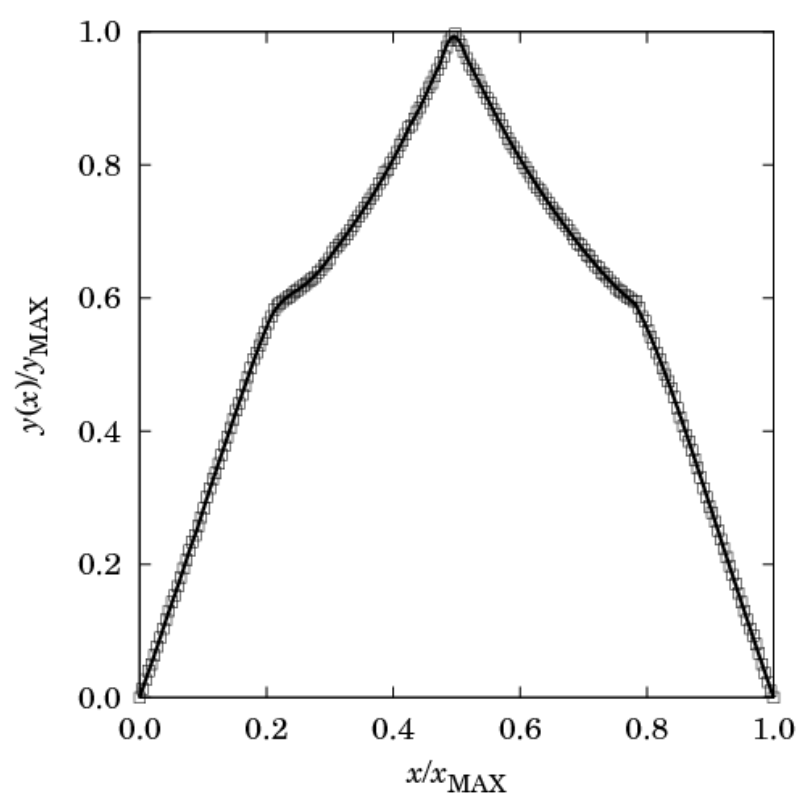

Figure 13 